\title{
The topology of the universe: the biggest manifold of them all
}

\author{
Janna Levin, Evan Scannapieco, and Joseph Silk \\ Center for Particle Astrophysics, UC Berkeley, Berkeley, CA 94720-7304
}

Clues as to the geometry of the universe are encoded in the cosmic background radiation. Hot and cold spots in the primordial radiation may be randomly distributed in an infinite universe while in a universe with compact topology distinctive patterns can be generated. With improved vision, we could actually see if the universe is wrapped into a hexagonal prism or a hyperbolic horn. We discuss the search for such geometric patterns in predictive maps of the microwave sky.

Contribution to the conference proceedings for the Cosmology and Topology Workshop to appear in Class. and Quant. Grav. 
The world abounds with objects that have multiconnected topology. The coffee cup comes to mind as the overused but adored example of a compact surface with genus 1 . There is also the coffee cup's topological equivalent, the donut. People, buildings, the Earth, are all topologically connected. As you begin to scan the examples you realize that every thing is at least finite if not actually multiply connected. By contrast, no thing is infinite. Yet cosmology has persisted in the assumption that the universe is infinite. Try explaining that to your friends and neighbors.

Ignoring the global topology of the universe is particularly neglectful in a theory that purports to be a theory of geometry. Einstein's revolution pivots on discarding the notion of a gravitational force in favor of a theory of geometry. Yet his theory is incomplete. While general relativity determines the local curvature of spacetime, it falls short of specifying the global topology. A complete theory of gravity should be able to predict topology [1]. In the absence of a theory of global geometry, we can still wonder how natural it would be to live in a given cosmos. In all, there are an infinite number of multiconnected topologies but only three simply connected ones. Consistent with the belief that we are not in a privileged place in the universe, we should not live in a geometrically special space. The proliferation of compact spaces, particularly hyperbolic, compact spaces [2], indicates that a compact universe may be more probable [3]. A quantum cosmological treatment is needed to make this notion more precise [4].5]. While it is difficult to define a measure on the set of compact spaces, it is at least clear that the assumption of an infinite universe has no basis on which to claim naturalness.

We can observe topology despite our inability to predict it. Granted, an era of inflation would likely push the size of the fundamental domain beyond the observable universe. However, inflation also predicts that the curvature radius is huge relative to the size of the observable universe. Since observational evidence is favoring a negatively curved space, theoretical prejudices are considerably weakened. If curvature is observable, then perhaps so too is topology.

Limits on a universal topology scale are often set by searching for periodicity in observations of large structures [6,7]. Ghost images of any source appear as light wraps around the universe along different paths and replicates its image for any observer. This leads to an extreme version of Obler's paradox whereby a single bright source could light up the sky if left shining for long enough [8]. On an astronomical scale, there would be ghosts of galaxies and quasars. Since the ghosts are not identical clones, but are rather images of the source at different ages, a search for ghosts is impeded by evolutionary effects and in the end may be a difficult way to identify the connectedness of space.

A far more sensitive probe is offered by the the cosmic background radiation (CBR). This one relatively unmarred fossil record from the early universe can reveal the large-scale landscape of the universe. Tiny fluctuations in the spacetime geometry appear as hot and cold spots in the otherwise smooth primordial radiation. The geometric fluctuations at the time light last scatters can be reconstructed from these hot and cold spots and offers a critical test of small topologies. Only perturbations that fit inside the compact manifold are allowed. The result is a pattern of fluctuations on the microwave sky reflecting the full structure of spacetime, local and global.

The challenge is to predict the spatial pattern given the geometry. We discuss the reflection of geometry in the CBR sky in flat topologies (乌) and in the one multiconnected (but not fully compact) hyperbolic space for which CBR maps have been constructed ( $\S$ [I] $)$. None of the fully compact hyperbolic geometries have been constrained and have in fact become the topic of much recent interest [1,3,9, 10. . We are learning from the simpler spaces how to predict the features in the microwave sky for the resistant compact hyperbolic cosmos (§III).

\section{COMPACT, FLAT COSMOLOGIES}

We want to predict a map of the temperature fluctuations. In a homogeneous and isotropic space, an angular average over the fluctuations contains all of the essential information. Of the six compact, orientable flat spaces, all destroy global isotropy and all except for the hypertorus destroy global homogeneity. As a result, there is more information in a map of temperature fluctuations than just the angular power spectrum. Although we argue that the angular average overlooks conspicuous features in general, for the equal sided flat cases angular spectra do provide a reasonable bound.

Four of the six orientable, compact topologies of $\mathbf{E}^{3}$ are constructed from a parallelepiped as the fundamental domain. The other two are built from a hexagonal prism. The hypertorus is the simplest and has been studied by many authors 66,11 13. Stevens, Scott, and Silk 12] pointed out that in a flat 3-torus, the spectrum of temperature fluctuations was truncated at long wavelengths in order to fit within the finite box. Contrary to standard lore, we find all of the equal sided compact flat manifolds show a truncation in the power of fluctuations on wavelengths comparable to the size of the fundamental domain [14,15]. The longest wavelength fluctuation observed, namely the quadrupole, is in fact low. Some might even take this as evidence for topology [16]. Cosmic variance is also large on large scales. Consequently, a fundamental domain the size of the observable universe is actually consistent with the COBE data [14].

A very small universe however is incompatible with the data. The cutoff in long wavelength perturbations is accompanied by gaps in power at wavelengths that do not correspond to integer windings through the fundamental domain. All compact spaces show discrete harmonics and as such the sharp harmonics may be a more generic sign 

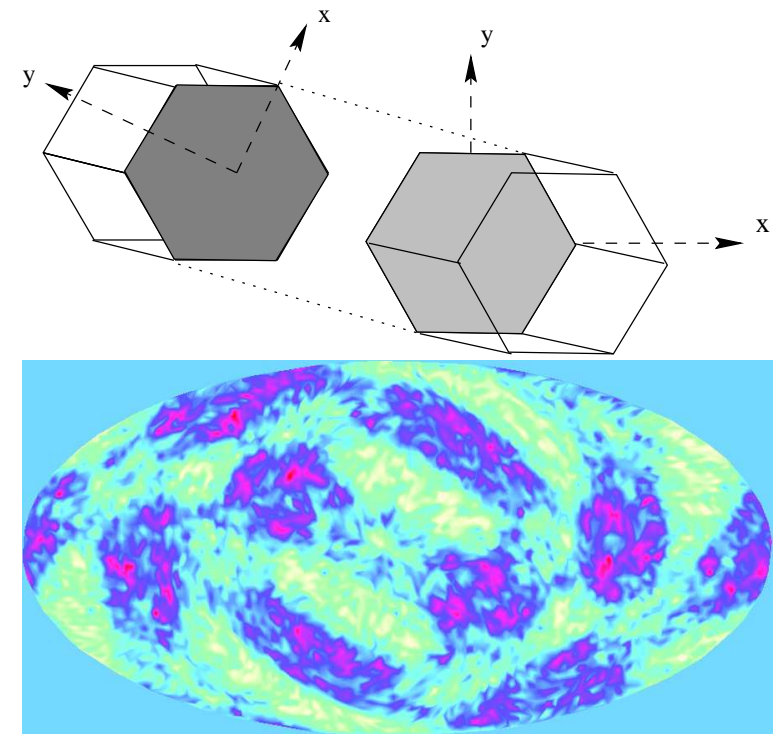

FIG. 1. A hexagonal prism with a $2 \pi / 3$ twist. The observer is at the center of the universe in the map of $\delta T(\hat{n}) / T$. The fundamental domain is half the diameter of the observable universe in two directions and one-tenth that in the twisted direction.

of compact topology. The jaggy spectra of such small compact flat spaces are tens of times less likely than the smooth spectrum of infinite $\mathbf{E}^{3}$. We conclude, quite conservatively, that the universe, if finite and flat and equalsided, must be at least $80 \%$ the radius of the surface of last scatter and so $40 \%$ of the diameter of the observable universe. There could still be as many as eight copies of our universe within the observable horizon.

If instead of an equal-sided space we consider a fundamental domain with disparate length scales, the angular power spectrum is in general a poor discriminant. The averaging over the sky fails to recognize the strong features in the cosmos. Fig. 11 shows a predictive map of the hot and cold fluctuations in a $2 \pi / 3$-twisted hexagonal prism. We have set the length of the fundamental domain to be ten times smaller in the twisted direction than along the face of the hexagon. The average large angle power in fluctuations is actually consistent with the data, although clearly this anisotropic space does not look like the sky we observe. A better statistic to discern patterns and correlations is badly needed [14,17]. The promising suggestions of [9, 3, 17] may be the key and are discussed more in $\S[$ III.

We could have predicted certain features of the map of Fig. 1, even if we had not known the eigenmodes explicitly. A $2 \mathrm{D}$ slice through the $3 \mathrm{D}$ tiling of space is represented in Fig. 2. If we draw bands connecting opposite sides of the hexagons and highlight any overlaps, we can predict the imprint of one mode as shown on the top of Fig. 2. Given that we do know the eigenmodes, we can show the actual contour plot of the hot and cold fluctuations for a similar mode on the bottom of Fig. 22. Comparing the guess with the actual contours shows our
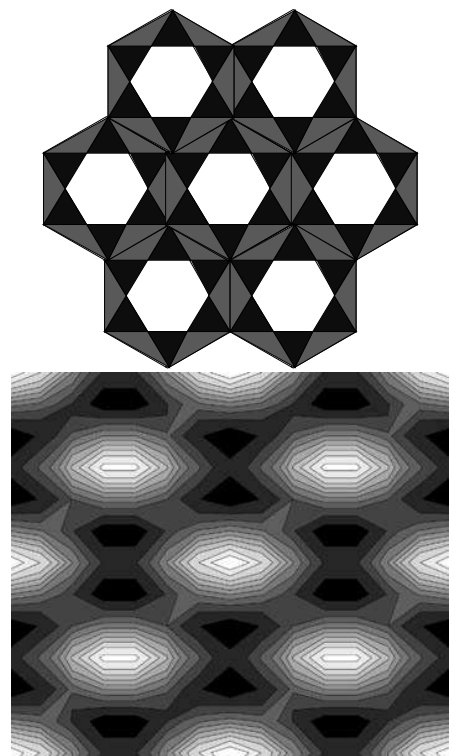

FIG. 2. Top: A guess at one mode. Bottom: A contour plot of the temperature fluctuation for a similar mode.

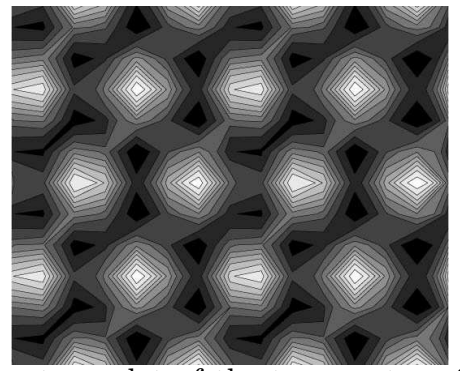

FIG. 3. A contour plot of the temperature fluctuation for a mode that winds through the twisted prism.

guess did quite well. The hexagonal shape of the universe is clearly seen. In actuality, there are many modes competing to imprint a pattern on the sky which blurs the signature hexagons. In Fig. 3, is another contour plot of hot and cold spots for a different mode which exhibits the $2 \pi / 3$ twist through the prism.

The competition between fluctuations obscurs some features while enhancing others. The surface of last scatter cuts a sphere out of the full 3D space, an elliptic projection of which is given in the map of Fig. 1. Can you see hexagons in the map? Almost. Is the $2 \pi / 3$ twist in the space visible? We are currently developing ways of looking at the sky that pull the underlying patterns out of the noise 17].

\section{MULTICONNECTED, HYPERBOLIC HORN}

No compact hyperbolic manifolds have yet been constrained. The only multiconnected hyperbolic space for which predictive maps have been constructed is the horn topology introduced by Sokolov and Starobinsky [18. The horn is not completely compact but is only closed 
off in two directions. A two dimensional subspace is wrapped into a flat torus. This torus is then conformally stretched or shrunk along the third dimension tracing out a horn (Fig. 4). There is no chaos on this space since it is only partly compact. We were thus able to find exact solutions for the geodesic motions and the perturbation spectrum. While the horn topology may seem a special case, there are many manifolds that may bear hornlike corners. As emphasized in [3], many manifolds have cusps. To an observer nested in this region the world looks very much like a toroidal horn.

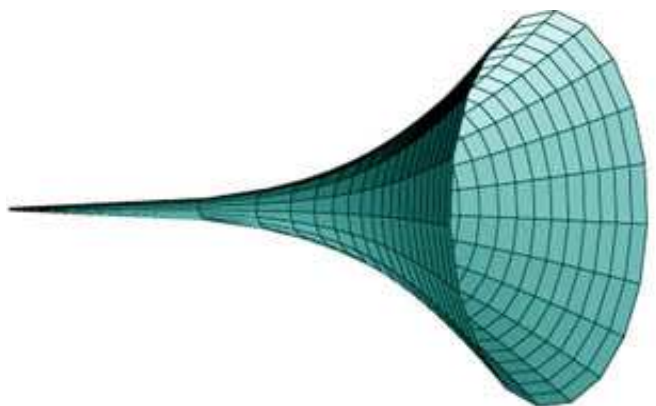

FIG. 4. An embedding diagram of the horn with one of the dimensions hidden.

In the narrow throat of the horn, big hot and cold spots cannot be supported and the temperature of the CBR looks smooth there as demonstrated in the simulated maps of Fig. 局. The upper panel shows a horn with topology scales around the observer equal to the radius of the last scattering surface in one direction and half that in the other compact direction. Notice that the observer can see exponentially deep down the narrowing throat of the horn. The bottom panel shows topology scales equal to $67 \%$ of the radius of the last scattering sphere in one compact direction and $10 \%$ in the other. Again, you can actually see the geometry of the horn [10].

As before, the averaged angular power spectrum is a weak criterion by which to search for topology. The horn is infinite if multiconnected. There is therefore formally no cutoff in the angular power spectrum. Long wavelength modes cannot fit in the two compact directions but infinitely long modes can exist along the axis of the horn. Again, the anisotropy and inhomogeneity would be washed out if we only considered the angular power spectrum and never looked at a map of the full $\delta T(\hat{n}) / T$. If the universe were completely compact and hyperbolic with cuspy corners, there may again be no formal cutoff in long wavelength power [3]. However, there may still be patches in the sky that were too small to support fluctuations and hence flat spots would appear.

Looking at the sky as seen by COBE, it does not appear that we live within view of any cuspy corners. It also appears that a pattern imprinted on the microwave sky will likely be more incisive in identifying topology than ghost images. The pattern may not bear a flat spot, but it will reflect the underlying symmetry group of the manifold.

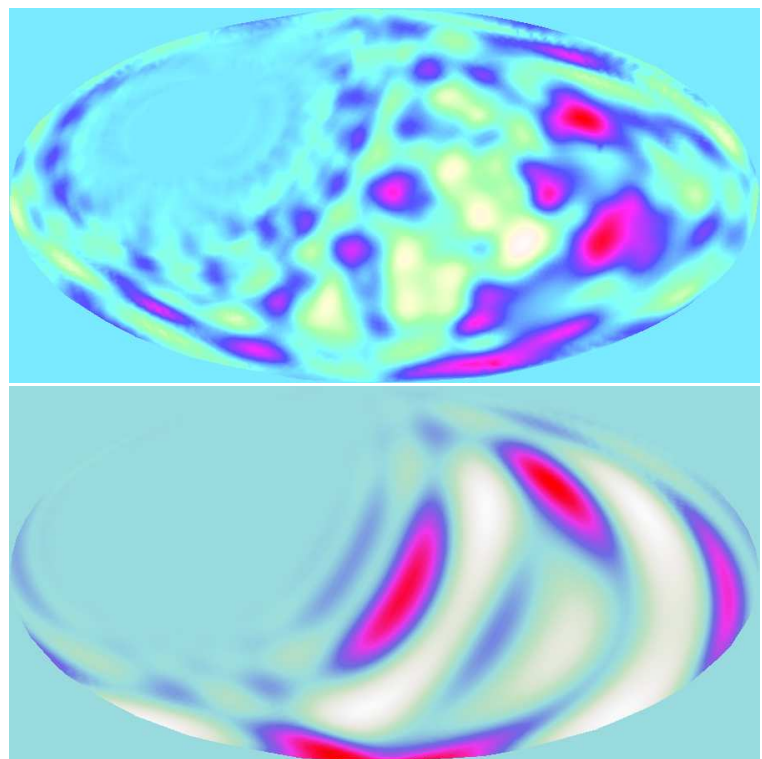

FIG. 5. The predicted COBE sky in a horn topology.

\section{COMPACT, HYPERBOLIC}

In the previous two sections we were able to predict the distribution of hot and cold spots across the sky given the geometry. We could do so because we could solve for the spectrum of temperature fluctuations explicitly. In a totally compact, hyperbolic space however, the spectrum cannot be obtained analytically. The inability to decompose fluctuations in the photon background is a direct consequence of the chaotic mixing on compact, negatively curved surfaces.

Techniques have been attempted in order to circumvent the eigenvalue problem. The correlation function has been computed using the method of images [9]. This method involves the sum of the correlation function in the universal covering space with ghost images in the copies. The temperature correlation function can then be compared with the COBE data. The method requires a detailed knowledge of the elements of the symmetry group. The sum is highly divergent and is difficult in itself to manage. The divergence results since the number of images proliferates at large distances.

Another approach focuses on the effect of topological lensing of the last scattering surface. A well planned statistic may be found which can determine if two rings in the sky are really copies of the same collection of points on the surface of last scatter [3, 19. High resolution and sensitivity are demanded of an experiment to observe the thin rings. The future satellites such as the Microwave Anisotropy Probe (MAP) and Planck are needed to discriminate a universe with such circles in its sky from one that is simply connected [20,3].

Another alternative is to emphasize the spatial patterns in the sky, although ultimately all the approaches are interwoven. In the previous examples, the universe clearly showed spatial patterns that exposed the under- 
lying geometry. By understanding the symmetries of the fundamental polyhedron and the identification rules, a CBR pattern can be deduced without the need to explicitly obtain the spectrum mode by mode [17]. Given a breakdown of the pattern, a method not unlike that suggested in [21] can be implemented to reconstruct the fundamental domain. A similar philosophy was used to search for the symmetry axes of a hypertorus in [13. No such axis of symmetry was detected, refining the bound on anisotropic hypertori.

Geometric patterns have also been used successfully to predict COBE maps for the Bianchi classes [22]. These Bianchi classes are directly related to Thurston's eight geometries [2]. In addition to the three of constant curvature, there are five more homogeneous but anisotropic spaces. The sky patterns can be predicted just from a knowledge of the group invariances that generate the geometries and their geodesic flows [23,24]. There is a physical difference here from the multiconnected models. For the Bianchi cosmologies, there are assumed to be no initial perturbations. The temperature fluctuations in the CBR result as the shear and expansion of the evolving geometry Doppler shift the photons while they transit the anisotropic space.

There is even precedence in biology. Spatial pattern formation emerges on the backs of mammals and in part answers the question of how the leopard got its spots [25. The enzymes responsible for the pigmentation on an animals fur fluctuate through the body. The geometry of the developing animal and its scale relative to the characteristic wavelength of the fluctuating enzymes differentiate the markings. The universe in its early stages of development similarly acquires markings. Again, the geometry and scale of the space relative to the characteristic wavelength of the fluctuations in the CBR can influence patterning. We have already seen the universe wear different coats in Figs. 11 and 5 reminiscent of a leopard's spots or a zebra's stripes. Surprisingly, biology is a cleaner system than cosmology since diffusion mechanisms often single out one mode and thereby one clean pattern. In cosmology, many modes are summed and so many patterns compete in making the universe's coat. This was evidenced in the hexagons of Fig. 11 versus those of Figs. 2 and 3. It is a theoretical prejudice that fundamental physics is simple and everything else is just a very messy composite. Still, the codes of nature replicate themselves on scales vast and small. It will be ironic if in this instance we have to first turn to biology to find the clues to the universe and perhaps even a fundamental theory of gravity.

As the observational evidence accumulates, we are forced to confront the very real possibility that the universe is negatively curved and that the curvature scale is just coming within view with an associated topology scale. If we do live in a compact, hyperbolic cosmos, we may have to rewrite the standard big bang story. A loose narrative for the history of the universe begins with the birth of the universe and implores an answer from quan- tum cosmology: Is the creation of compact, hyperbolic 3-manifolds favored over others? Once such a universe is born, chaotic mixing on the small space could lead to the relatively smooth cosmos observed today and has already been suggested as an alternative to inflation [26]. In fact, it is precisely this suggestion which spurred some searches for a small universe. While chaotic mixing could explain the average smoothness, the geometry might explain the local clumpiness of matter. Pattern formation due to geometry can affect the distribution of galaxies as well as the CBR [18,10]. The global topology may then create local inhomogeneity and distinctive patterns in the large-scale distribution of luminous and dark matter. The geometry may lead to bubbly or even fractal distributions of galaxies. This scenario for the evolution of our universe is compelling if optimistic. Perhaps geometry alone, and hence the theory of gravity, determines the birth and fate of the cosmos.

\section{ACKNOWLEDGMENT}

We have benefited from valuable discussions with nearly everyone currently active in the area of topology and cosmology. We are grateful to all of these collaborators and colleagues: J. Barrow, J. Bond, R. Brooks, N. Cornish, P. Ferreira, D. Pogosyan, T. Saurodeep, D. Spergel, G. Starkman, W. Thurston and J. Weeks. Special thanks to all of the enthusiastic participants of the workshop.

[1] M. Lachieze-Rey and J.P. Luminet, Phys. Rep. 254 (1995) 135.

[2] W.P. Thurston, Bull. Am. Math. Soc. 6 (1982) 357; W.P. Thurston and J.R. Weeks, Sci. Am. July (1984) 94; W.P. Thurston, "Three-dimensional geometry and topology" (Ed: Silvio Levy, Princeton University Press, Princeton, N.J. 1997).

[3] N.J. Cornish, D. Spergel and G. Starkman, astroph/9602039 (1996); ibid. astro-ph/9708225 (1997).

[4] G. W. Gibbons and M. J. Perry, Nucl. Phys. B146, 90 (1978).

[5] S. Carlip, "Spacetime Foam and the Cosmological Constant", gr-qc/9708026.

[6] D.D. Sokolov and V.F. Shvartsman, Sov. Phys. JETP 39, (1975) 196; G. Paal, Acta. Phys. Acad. Scient. Hungaricae 30, (1971) 51; J.R. Gott, Mon. Not. R. Astron. Soc. 193 (1980) 153.

[7] B.F. Roukema and V. Blanloeil, this volume.

[8] Comments on Obler's paradox grew out of conversations with J.D. Barrow.

[9] J. R. Bond, D. Pogosyan and T. Souradeep, preprint astro-ph/9702212 (1997); ibid. in preparation. 
[10] J. Levin, J.D. Barrow, E.F. Bunn and J. Silk, Phys. Rev. Lett. 79 (1997) 974.

[11] I.Yu. Sokolov, JETP Lett. 57 (1993) 617; A. de OliveiraCosta and G.F. Smoot Ap.J. 448 (1995) 447.

[12] D. Stevens, D. Scott and J. Silk, Phys. Rev. Lett. 71 (1993) 20.

[13] A.A. Starobinsky, JETP Lett. 57 (1993) 622; A. de Oliveira-Costa, G.F. Smoot and A.A Starobinsky, Ap.J. 468 (1996) 457.

[14] J. Levin, E. Scannapieco and J. Silk, "Is the universe infinite or is it just really big?", preprint CfPA-98-TH01, astro-ph/9802021.

[15] The CITA group was also aware that the longest modes were forbidden for some of the twisted flat spaces. T. Saurodeep, private communication.

[16] The low quadrupole sparked some of the recent interest in small universes as discussed at the workshop. Private communication G. Starkman and D. Spergel.

[17] J. Levin, E. Scannapieco, J. Silk and J.D. Barrow, "How the Universe Got Its Spots", in preparation.

[18] D. D. Sokolov and A. A. Starobinskii, Sov. Astron. 19 (1976) 629.

[19] N.J. Cornish, D. Spergel and G. Starkman, this volume.

[20] M. Kamionkowski and D. Spergel, Ap. J. 432 (1994) 7.

[21] J. Weeks, this volume.

[22] L. Bianchi, Mem. Soc. It. della Sc., Dei XL, (3), 11, 267 (1897); R. Kantowski and R.K. Sachs, J. Math. Phys. 7, 443 (1966).

[23] J.D. Barrow, R. Juszkiewicz and D.H. Sonoda, Nature 316, 48 (1985); J.D. Barrow, R. Juszkiewicz and D.H. Sonoda, Mon. Not. Roy. astr. Soc. 213, 917 (1985)

[24] J.D. Barrow and J. Levin,Phys. Lett. A 233 (1997) 169.

[25] J.D. Murray, Mathematical Biology (Springer-Verlag, New York, 1989).

[26] G. Ellis and R. Tavakol in "Deterministic Chaos in General Relativity" (eds: D. Hobill et. al., Plenum Press, NY 1994); C.M. Lockhart, B. Misra and I. Prigogne, Phys. Rev. D25 (1982) 921; V.G. Gurzdyan and A.A. Kocharyan, Astron. Astrophys. 260 (1992). 